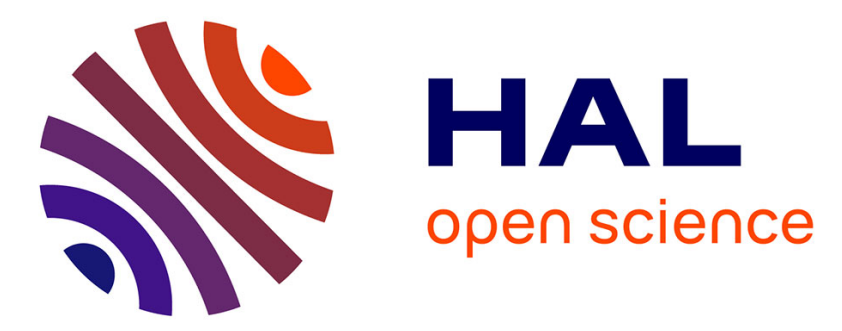

\title{
Can magneto-plasmonic nanohybrids efficiently combine photothermia with magnetic hyperthermia?
}

Ana Espinosa, Mathieu Bugnet, Guillaume Radtke, Sophie Neveu, Gianluigi

A. Botton, Claire Wilhelm, Ali Abou-Hassan

\section{- To cite this version:}

Ana Espinosa, Mathieu Bugnet, Guillaume Radtke, Sophie Neveu, Gianluigi A. Botton, et al.. Can magneto-plasmonic nanohybrids efficiently combine photothermia with magnetic hyperthermia?. Nanoscale, 2015, 7, pp.18872-18877. 10.1039/C5NR06168G . hal-01219175

\section{HAL Id: hal-01219175 \\ https://hal.sorbonne-universite.fr/hal-01219175}

Submitted on 26 Oct 2015

HAL is a multi-disciplinary open access archive for the deposit and dissemination of scientific research documents, whether they are published or not. The documents may come from teaching and research institutions in France or abroad, or from public or private research centers.
L'archive ouverte pluridisciplinaire HAL, est destinée au dépôt et à la diffusion de documents scientifiques de niveau recherche, publiés ou non, émanant des établissements d'enseignement et de recherche français ou étrangers, des laboratoires publics ou privés. 


\title{
Can magneto-plasmonic nanohybrids efficiently combine photothermia with magnetic hyperthermia?
}

\author{
Ana Espinosa, ${ }^{a}$ Mathieu Bugnet, ${ }^{b}$ Guillaume Radtke, ${ }^{c}$ Sophie Neveu, ${ }^{d}$ Gianluigi A. Botton, ${ }^{b}$ Claire \\ Wilhelm* $\ddagger^{\mathrm{a}}$ and Ali Abou-Hassan* $\ddagger^{d}$
}

\begin{abstract}
Multifunctional hybrid-design nanomaterials appears to be a promising route to meet current therapeutics needs required for efficient cancer treatment. Herein, two efficient heat nanogenerators were combined into a multifunctional single nanohybrid (a multi-core iron oxide nanoparticle optimized for magnetic hyperthermia, and a gold branched shell with tunable plasmonic properties in the NIR region, for photothermal therapy) which impressively enhanced heat generation, in suspension or in vivo in tumours, opening up exciting new therapeutic perspectives.
\end{abstract}

Nanomedicine has emerged as a rapidly developing field to face current diagnostics and therapeutics challenges. ${ }^{1}$ On the one hand, nanoparticles can be activated by a physical stimulus only once it has reached its target, thus preventing damaging effects on healthy bystander tissues, and on the other hand, several functions can be incorporated within a single nanostructure, to provide a synergic efficiency by merging multiple therapeutic modalities. ${ }^{2}$

One promising remotely activated nano-therapy is based on the ability of some nanoparticles to generate heat under external stimulus. ${ }^{3}$ Currently, gold (plasmonic) and iron oxide (magnetic) nanoparticles are the prime candidates for the development of such thermo-therapy. Optical excitation of plasmonic nanoparticles by an infrared laser causes localized heating that can destroy surrounding cancer cells (photothermia). ${ }^{4}$ In the case of magnetic nanoparticles, application of a high-frequency alternating magnetic field excites the fluctuations of the magnetic moment, the resulting magnetic energy being released and dissipated as heat (magnetic hyperthermia). In both cases, the size and shape of the nanoparticles are crucial. ${ }^{5}$ Indeed, gold nanoparticles such as gold nanoshells, ${ }^{6}$ nanostars, ${ }^{7}$ and nanorods ${ }^{8}$ are often preferred due to their strong absorption cross sections. Furthermore, the possibility

\footnotetext{
Laboratoire Matière et Systèmes Complexes (MSC), UMR 7057, CNRS and Université Paris Diderot, 75205 Paris cedex 13, France. E-mail: claire.wilhelm@univ-paris-diderot.fr.

b. Department of Materials Science and Engineering and Canadian Centre for Electron Microscopy, McMaster University, 1280 Main street West, Hamilton, ON, Canada L8S4M1.

- Institut de Minéralogie, de Physique des Matériaux et de Cosmochimie (IMPMC), UMR 7590, CNRS, UPMC, 4 place Jussieu, 75005 Paris, France.

${ }^{d .}$ Sorbonne Universités, Physicochimie des Electrolytes et Nanosystèmes InterfaciauX (PHENIX), UMR 8234, Université Pierre et Marie Curie UPMC-CNRS, 75252 Paris cedex 05, France.E-mail:ali.abou_hassan@upmc.fr. tElectronic Supplementary Information (ESI) available: [details of any supplementary information available should be included here]. See DOI: $10.1039 / x 0 x x 00000 x$.
}

¥These authors contributed equally to this work. of engineering their shapes results in surface plasmon resonances in the near infra-red (NIR) region, where light absorption by biological tissues is minimal (650-900 nm). ${ }^{9}$ For magnetic nanoparticles, finetuning the shape, magnetization and anisotropy is necessary to enhance the heat-generating capacity. New generation of magnetic cubic, $^{10}$ cooperative multicores, ${ }^{11}$ or core-shell nanostructures ${ }^{12}$ have recently reached unprecedented heating capacities.

Nanoscale combination of materials with magnetic and plasmonic properties has drawn particular interest these past few years. ${ }^{13}$ Several novel developments and recent reports in magnetoplasmonic multifunctional platforms have demonstrated a synergistic potential resulting from the association of both materials. ${ }^{14}$ For example iron oxide core nanoparticles with a gold shell have been synthesized to enable molecular optical and magnetic resonance imaging (MRI) in combination with gold photothermal therapy of cancer cells. ${ }^{14 a}$ Nano-transducers with a superparamagnetic iron oxide core embedded in a silica and a gold nanoshell have been studied in magnetic targeting and MRI detection imaging of brain tumors associated with photothermal ablation treatment provided by the gold nanoshell. ${ }^{14 \mathrm{~g}}$ These recent developments in magneto-plasmonics have thus mainly focused on the use of iron oxide cores for MRI, magnetic manipulation and targeting, while gold layers were integrated to provide optical response of the hybrids for imaging or photothermal heating. Combining efficiently the magnetic hyperthermia potential of iron oxide cores and the plasmonic photothermal effects of gold layers remains, however, an open challenge. Moreover, beside the bimodality in hyperthermia which could be brought by the combination of both materials in one single nanostructure, magneto-plasmonic nanohybrids allow to guarantee a similar biodistribution of iron oxide and gold in tissues, ${ }^{15}$ a rich surface chemistry for conjugation due to gold, compared to the iron oxide core alone, ${ }^{16}$ and finally a protective shield against the degradation of magnetic cores is brought by the gold shell. ${ }^{17}$ Up to date, magneto-plasmonic hybrids efficient for both magnetic hyperthermia and plasmonic photothermia are rare, ${ }^{18}$ and mapping of plasmons at the nanoscale, on an iron oxide core, is still unmet. Herein, we propose an efficient class of magneto-plasmonic nanoparticles (MagPlasNPs), which combine high thermal capabilities for both magnetic hyperthermia and photothermia at low excitations doses. We selected two high-performance heat nano-generators, a multi-core iron oxide nanoparticle for the magnetic part and a gold-branched nanostructure for the plasmonic part. Combined elemental and surface plasmon mapping performed at the nanoscale on single nanohybrids allowed for an unprecended assessment of plasmons on an iron oxide core, and the combination 
of magnetic and plasmonic properties in the single nanohybrids impressively enhanced heat generation, in suspension or in vivo in tumours.

The synthesis of MagPlasNPs was carried out using a seed-mediated growth approach as illustrated in Fig. 1a (see experimental section in Supplementary Information (SI) for full details). ${ }^{19}$

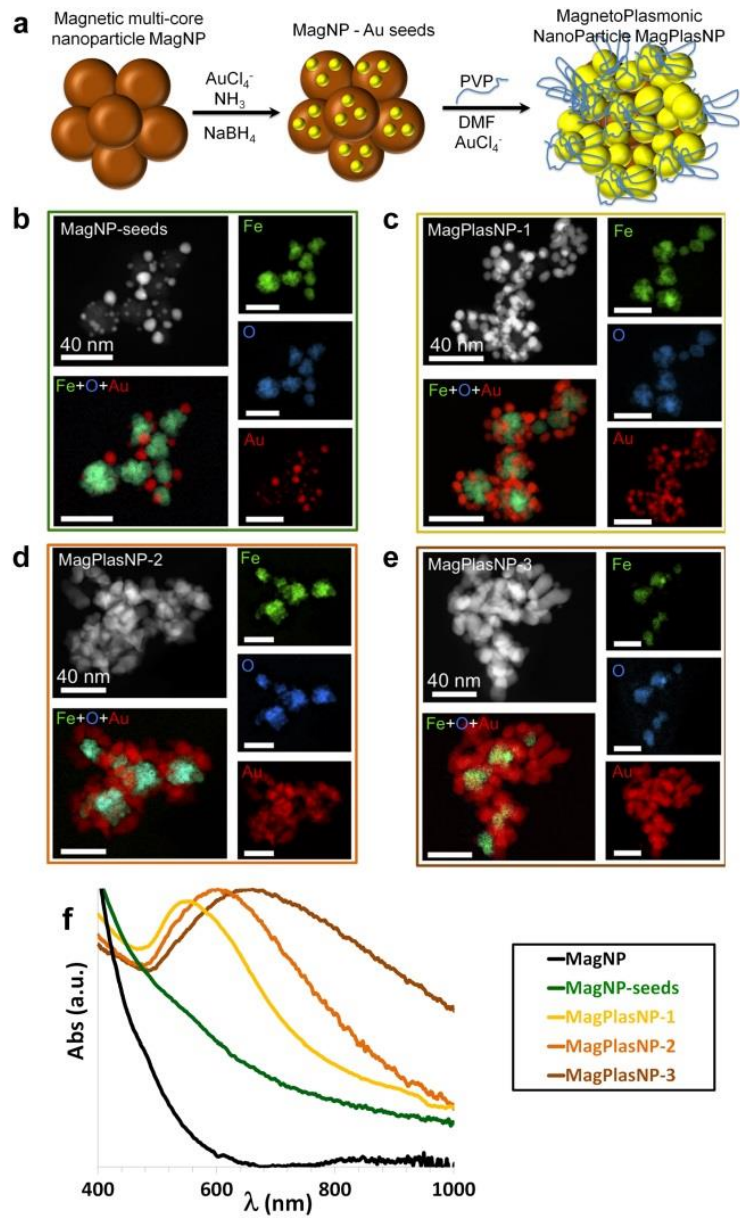

Fig. 1 (a) The seeding-growth process used for the synthesis of the magneto-plasmonic nanoparticles (MagPlasNP) of different ratio Au/Fe: MagPlasNP-1 (Au/Fe $=1.13 \pm 0.04$ ), MagPlasNP-2 (Au/Fe $=3.1 \pm 0.7)$ and MagPlasNP-3 (Au/Fe $=4.7 \pm 0.9)$. (b-e) STEM-EELS analysis of nanohybrids. HAADF image, EELS Fe elemental map (green), EELS O elemental map (blue), EELS Au elemental map (red) and overlaid elemental maps are shown for (b) MagNP-seeds, (c) MagPlasNP-1, (d) MagPlasNP-2, and (e) MagPlasNP-3. (f) Vis-NIR absorption spectra of the different structures. PVP: polyvinylpyrolidone DMF: dimethylformamide.

Maghemite multi-core shape nanoparticles (MagNPs) (average diameter $30 \pm 4 \mathrm{~nm}$ from TEM, see Fig. S1) were synthetized as cores for the magneto-plasmonic hybrids and they were prepared via the polyol process. ${ }^{20}$ Afterward, their surface was functionalized with citrate anions which guaranteed for the seeding step a high colloidal stability in ammonia solution $(\zeta=-80 \mathrm{mV}$ at $\mathrm{pH}=10)$ and efficient attraction of $\left[\mathrm{Au}\left(\mathrm{NH}_{3}\right)_{4}\right]^{3+}$ that was reduced in the following step into Au seeds with $\mathrm{NaBH}_{4}{ }^{21}$

The resulting magnetic nanoparticles decorated with $\mathrm{Au}$ seeds (MagNP-seeds) were then redispersed in ethanol in presence of polyvinylpyrolidone (PVP) to enable the growth of spiked or multibranched gold shells during the step in presence of dimethylformamide (DMF), PVP and $\mathrm{AuCl}_{4}^{-}$(see experimental section in $\mathrm{SI}$ for more details). ${ }^{22}$ Transmission electron microscopy images (TEM) are shown in Fig. S1 for MagNP-seeds, with average diameter, $\mathrm{d} \approx 39 \pm 4 \mathrm{~nm}$, MagPlasNP-1, $\mathrm{d} \approx 47 \pm 6 \mathrm{~nm}$, MagPlasNP-2, $\mathrm{d} \approx 53 \pm 7 \mathrm{~nm}$ and MagPlasNP-3, $\mathrm{d} \approx 63 \pm 11 \mathrm{~nm}$.

Scanning transmission electron microscopy (STEM) was also employed to obtain the chemical images of the different magnetoplasmonic nanohybrids at the seed stage (MagNP-seeds), and after their growth under different synthesis conditions to final MagPlasNPs. In each case, high-angle annular dark field (HAADF) images are shown together with the chemical maps recorded simultaneously using electron energy loss spectroscopy (EELS) in Fig. 1 (see also Fig. S2 and S3). As the intensity scattered by an atom in HAADF roughly scales as $Z^{1.7}$ where $Z$ is the atomic number, ${ }^{23}$ the Au seeds formed after the seeding step appear much brighter than the maghemite cores. The $\mathrm{Fe}, \mathrm{O}$ and $\mathrm{Au}$ elemental maps shown together in Fig. 1b confirm this chemical contrast and the desired complex architecture. Also, the attachment of $\mathrm{Au}$ seeds to the surface of MagNPs resulted in the appearance of a plasmonic shoulder at $\lambda \sim 530 \mathrm{~nm}$ originally absent in the spectrum of MagNPs (Fig. 1f). This result is expected for discrete gold clusters on iron oxide cores, ${ }^{24}$ and is in agreement with the observed brown colour of the MagNP-seeds in ethanol. After the growth step, depending on the ratio of $\mathrm{HAuCl}_{4}$ to MagNPs available during the growth, we observed different organizations and thicknesses of the Au shells. For a final ratio of $\mathrm{Au} / \mathrm{Fe}=1.13 \pm 0.04$ (MagPlasNP-1, Fig. 1c) the size of the seeds increased from $\sim 4 \mathrm{~nm}$ to $\sim 10 \mathrm{~nm}$ in diameter. Moreover, individual quasi-spherical gold nanoparticles homogeneously distributed on the surface of maghemite cores with an interparticle distance of 10 to $15 \mathrm{~nm}$ can be observed. For the MagPlasNP-2, characterized by a $\mathrm{Au} / \mathrm{Fe}=3.1 \pm 0.7$ ratio, a gold shell of thickness reaching 15 to $20 \mathrm{~nm}$ around the maghemite cores is observed, forming a discontinuous thick shell as highlighted in the HAADF image and corresponding chemical maps in Fig. $1 \mathrm{~d}$. Moreover, the anisotropic shape of the gold particles forming the shell is more pronounced and spikes are observed. Finally, for the MagPlasNP-3, characterized by a $\mathrm{Au} / \mathrm{Fe}=4.7 \pm 0.9$ ratio, the gold shell thickness increased to more than $20 \mathrm{~nm}$, so that maghemite cores can barely be detected in the core due to the very efficient coverage of the gold shell (Fig. 1e). As the concentration of $\mathrm{HAuCl}_{4}$ in the growth step increased, the interparticle space still present at the maghemite surface for MagNP-seeds and MagPlasNP-1 disappeared completely in MagPlasNP-2 and MagPlasNP-3, in favor of a continuous spiky shell formed by merging gold nanoparticles of larger sizes. These results are in excellent agreement with conventional TEM observations (Fig. S1) and with the results obtained by UV-Vis-NIR spectroscopy (Fig. 1f). Indeed the suspension became blue and the resonance, red-shifted indicating growth and merging of Au seeds into spiky shells. Such shift was reported by other groups after growth of Au shells on magnetite iron oxide cores. ${ }^{2 a}{ }^{25}$ Beside the optical properties in the NIR, the superparamagnetic behavior of the iron oxide core remains unmodified by the gold shell (Fig. S4). Finally, the mean hydrodynamic diameters of all NPs were measured by dynamic light scattering (DLS, Fig. S5) and by recording their Brownian motions (Nanosight, Fig. S6). It increased from MagNPs to MagPlasNP-3, and was found higher than the TEM measurements due to the hydration of the multi-layered PVA, ${ }^{26}$ but never exceeding $200 \mathrm{~nm}$, revealing the absence of any aggregation. The colloidal stability of the MagNPs and MagPlasNPs after dispersion in physiological media (phosphate buffer solution (PBS), fetal bovine serum (FBS) and plasma) was also checked using DLS (Fig. S7). While citrated MagNPs quickly destabilized, MagPlasNPs showed a remarkable 
stability evidencing the role of the PVP coating.The plasmonic response of isolated MagPlasNP-2 hybrids was then investigated at the nanoscale using STEM-EELS (Fig. 2). ${ }^{27}$
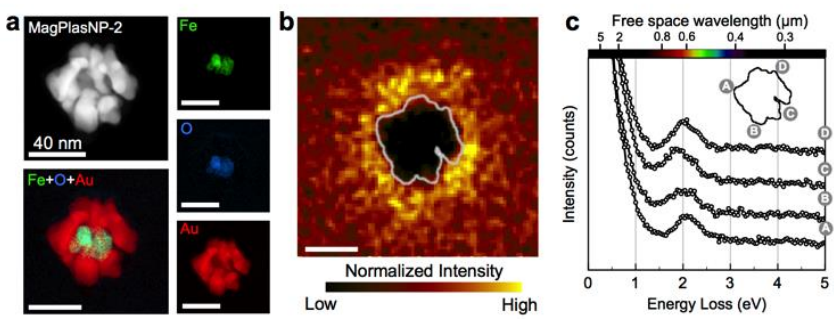

Fig. 2 Surface plasmon resonance of a single nanohybrid MagPlasNP-2. (a) High-angle annular dark field (HAADF) image and electron energy loss spectroscopy (EELS) elemental maps of a single nanohybrid (MagPlasNP-2), confirming the presence of an iron oxide core of $\sim 30 \mathrm{~nm}$ in diameter covered with a $\sim 20 \mathrm{~nm}$ thick discontinuous $\mathrm{Au}$ shell. (b) Surface plasmon resonance map (smoothed) corresponding to the 1.4-2.4 eV energy range. The contour of the nanohybrid is superimposed to the map. (c) EELS corresponding to four different beam positions around the nanohybrid. A single broad resonance is observed at $\sim 2.0 \mathrm{eV}(\lambda \sim 620 \mathrm{~nm})$ is observed. Raw data are shown.

The EELS map shown in Fig. $2 b$, corresponding to the integrated intensity in the 1.4-2.4 eV energy range highlights a localized surface plasmon resonance around the nanohybrid. The raw spectra displayed in Fig. $2 \mathrm{c}$ correspond to different positions of the electron beam around the nanohybrid as indicated in the sketch in the inset. A broad surface plasmon resonance is consistently observed at $\sim 2.0+/-0.2 \mathrm{eV}(\lambda \sim 620 \mathrm{~nm})$. Slight shifts of the surface plasmon resonance, such as the one observed in position $C$, can be assigned to the local variations of the shape of the $\mathrm{Au}$ nanocrystals forming the shell. The different assemblies of nanohybrids observed display a sizeable dispersion in their surface plasmon resonance energies, ranging from the visible $(\lambda \sim 560 \mathrm{~nm})$ to the near infrared $(\lambda \sim 855 \mathrm{~nm})$ regions, as well as in the number of distinguishable modes (see also Fig. S8 and S9). These results, obtained on single or multi-maghemite core nanostructures, establish an unambiguous relation between their nanoscale and macroscopic optical properties. Indeed, the typical optical response of an isolated MagPlasNP-2 nanostructure consists primarily in a surface resonance at about $620 \mathrm{~nm}$, confirming accurately the UVVis-NIR spectroscopy observations shown in Fig. 1f. Furthermore, intrinsic size and shape inhomogeneities combined with the formation of anisotropic agglomerates of multi-core nanostructures lead to slight energy shifts of the surface plasmon, and even to the appearance of multiple modes explaining the sizeable energy broadening of the resonance observed in the macroscopic measurements.

Magneto-plasmonic nanohybrids were then subjected to magnetic and laser stimulations. First, all nanohybrids were dispersed at [Fe] $=7 \mathrm{mM}$ in water, and exposed to i) magnetic hyperthermia (MHT) at magnetic field of $900 \mathrm{kHz}$ and $25 \mathrm{mT}$, (ii) $680-\mathrm{nm}$ NIR laser at a power density of $0.3 \mathrm{~W} / \mathrm{cm}^{2}$ (PLASM), and (iii) combined magnetic field and laser at the exact same conditions (MHT+PLASM) (Figs. 3a and $3 \mathrm{~b}$ ). In the first case (MHT only), the same temperature elevation of $6^{\circ} \mathrm{C}$ was observed for all samples, corresponding to a heating power (specific absorption rate (SAR) expressed in W/g of iron) at $634 \pm 76 \mathrm{~W} / \mathrm{g}$, in the range of the highest SAR yet reported. This excellent magneto-thermal efficiency is brought by the multicore assembly which decreases the surface disorder and enhances the magnetic susceptibility, thus potentiating thermal losses. ${ }^{11}$ During laser exposure at $0.3 \mathrm{~W} / \mathrm{cm}^{2}$, the temperature elevation was tuned by the nanohybrid design, increasing from $2^{\circ} \mathrm{C}$ for MagNPs, to $6^{\circ} \mathrm{C}$ for MagPlasNP-1 and $11^{\circ} \mathrm{C}$ for MagPlasNP-2 and 3. The combination of both magnetic and laser exposures (MHT+PLASM) led to a remarkable accumulation effect, reaching $14^{\circ} \mathrm{C}$ elevation for MagPlasNP-1, and up to $18-19^{\circ} \mathrm{C}$ for MagPlasNP-2 and 3 (see also the cumulative effect for a $808 \mathrm{~nm}$-laser excitation in Fig. S10, and the influence of laser power density, from 0.1 to $0.5 \mathrm{~W} / \mathrm{cm}^{2}$ in Fig. S11). Second, the MagPlasNP-2 heat generation potential was investigated as function of the concentration ( $[\mathrm{Fe}]=3.5,7,15,30$ and $60 \mathrm{mM}$ ) (Figs. 3c-d), using a magnetic coil ensuring biosafety for future clinical application $(110 \mathrm{kHz}, 25 \mathrm{mT})$. The magnetic heating was proportional to the concentration, corresponding to a heating power of $170 \pm 30 \mathrm{~W} / \mathrm{g}$ (consistent with the decrease in frequency to $110 \mathrm{kHz}$ ). The $680 \mathrm{~nm}$ laser was used at the same low power $\left(0.3 \mathrm{~W} / \mathrm{cm}^{2}\right)$, evidencing a saturation of the heating capacity with concentration. In the magneto-plasmonic mode, the resulting thermal efficiency increased with the concentration, and therapeutic temperature elevations over $17^{\circ} \mathrm{C}$ were achieved in very short times ( $100 \mathrm{~s}$, see Fig. S12 for the temperature profiles) for concentrations in the $[\mathrm{Fe}]=30-60 \mathrm{mM}$ range.

The combined heating was finally tested in a mouse animal model (Fig. 3e) in order to evaluate its workability in vivo. Before injecting in animals, we determined that the nanohybrids were biocompatible and effective for tumour cell capture (Fig. S13). This biocompatibility, confirms the long-standing and safe record of PVP coating in biomedical/pharmaceutical applications. ${ }^{28}$ The thermal window to achieve tumor ablation corresponds to $45-48^{\circ} \mathrm{C}$ temperature range. For subcutaneous tumor (initial temperatures around $28^{\circ} \mathrm{C}$ ), it means a temperature elevation of $17-20^{\circ} \mathrm{C}$. For a tumor volume of about $150 \mathrm{~mm}^{3}$, we thus chose to inject intratumorally $50 \mu \mathrm{l}$-suspension of MagPlasNP-2, at $[\mathrm{Fe}]=150 \mathrm{mM}$ (expected final intratumoral concentration in the $50 \mathrm{mM}$ range, corresponding to a potential $18-20^{\circ} \mathrm{C}$ temperature elevation). The collateral tumor was non-injected and considered as control. Both magnetic induction and laser irradiation alone showed an efficient and rapid thermal performance with a $9-10^{\circ} \mathrm{C}$ temperature increase, while no temperature increase was observed in noninjected tumor. Under the two stimulations, the heating was definitely cumulative, with an increase of almost $20^{\circ} \mathrm{C}$, reached within $2 \mathrm{~min}$. Moreover, the heating performance was still high after 3 days $(80 \%$ of heating maintained), indicating lasting effectiveness (Fig. S14). Histological sections were examined for a tumor excised after injection, revealing nanohybrids-rich zones around the tumor corona that diffusely penetrate to deeper tissues (Fig. S15), confirmed as well by TEM observations (Fig. S16) close and far to the injection spot. Nevertheless, the tumoral localization of the nanohybrids was sufficient to afford global heating in macroscopic zones, even 2 days after injection (see Fig. S14).

Finally, a preliminary follow-up of the tumor growth was performed after the combined treatment, revealing a promising therapeutic tumor regression during the 5 following days, in comparison with the control tumors (Fig. S17) 
a

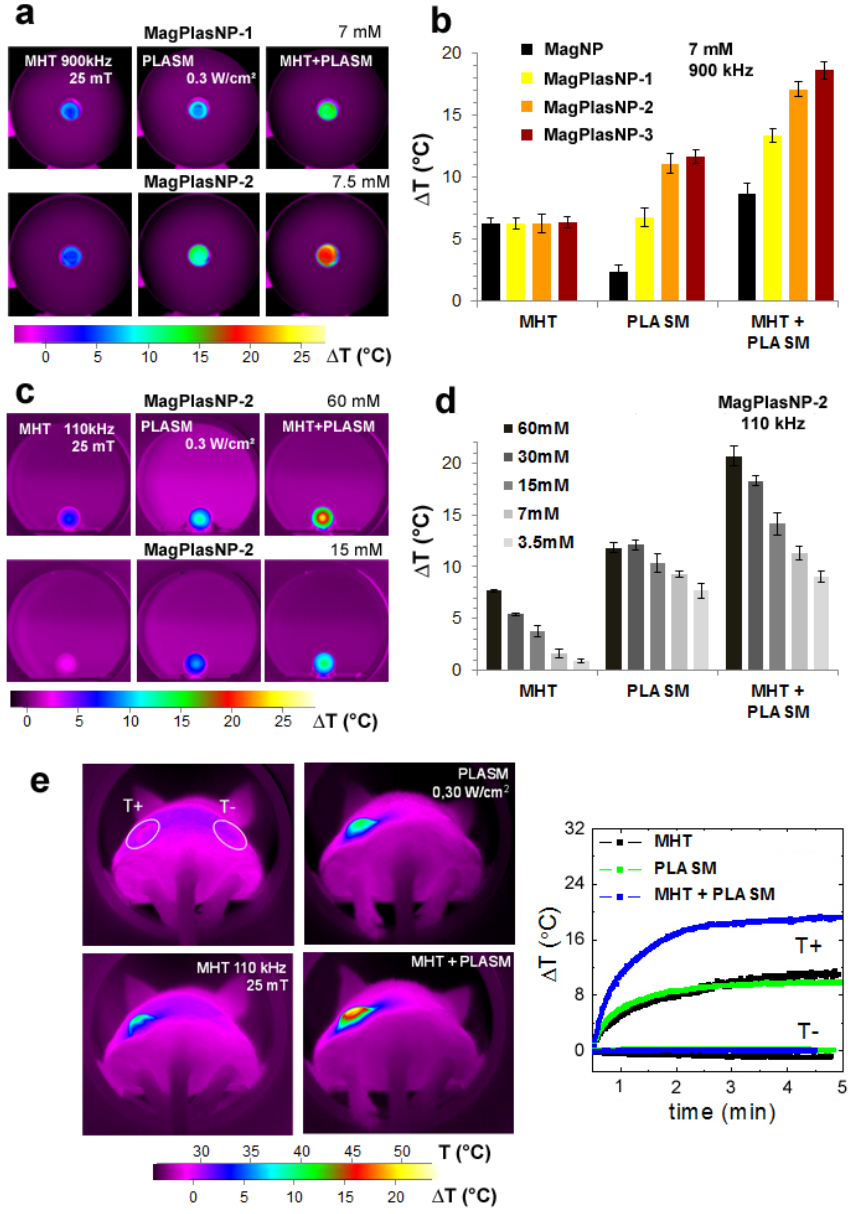

Fig. 3 Heating efficiency of the nanohybrids. $(a, b)$ : Tunability of the heat generation with the nanohybrids design. All NPs were dispersed at the same concentration $[\mathrm{Fe}]=$ $7 \mathrm{mM}$. The magnetic coil used delivered a $(900 \mathrm{kHz}, 25 \mathrm{mT})$ magnetic field. $680 \mathrm{~nm}$ laser was used at $0.3 \mathrm{~W} / \mathrm{cm}^{2}$. (a): Typical infrared images after 5 min heating of magneto-plasmonic nanoparticles (MagPlasNP-1 and MagPlasNP-2) under magnetic hyperthermia (MHT), laser irradiation (PLASM) and the combination of both modalities (MHT + PLASM) (100 $\mu \mathrm{l} \mathrm{sample} \mathrm{in} 500 \mu \mathrm{l}$ eppendorf ( $8 \mathrm{~mm}$-diameter) placed within the coil). (b): Average temperature elevation recorded for magnetic (MagNPs) and magneto-plasmonic nanoparticles (MagPlasNP-1, MagPlasNP-2 and MagPlasNP-3) under the three different heating protocols $(c, d)$ : Tunability of the heat generation with the nanohybrids (MagPlasNP-2) concentration. MHT was now performed in conditions of biosafety (magnetic coil at $110 \mathrm{kHz}$ frequency). Same laser settings were used (680 $\mathrm{nm}, 0.3 \mathrm{~W} / \mathrm{cm}^{2}$ ). (c): Typical infrared images after 5 min heating for MagPlasNP-2 at [Fe $]=15$ and $60 \mathrm{mM}(100 \mu \mathrm{l}$ sample in a home-made $8 \mathrm{~mm}$ tube containing a cylindrical hole of $3 \times 3 \times 5 \mathrm{~mm}$ to be filed with the solution). (d): Average temperature elevations recorded in all conditions. The magnetic heating (MHT) increases linearly with the concentration, while the laser efficiency (PLASM) saturates at high concentrations. As a result, the magneto-plasmonic heating (MHT+PLASM) mostly follows the magnetic-related variation with concentration. (e): In vivo hyperthermia performance. A solution of $[\mathrm{Fe}]=150 \mathrm{mM}$ of MagPlasNP-2 is injected into the tumour $(T+)$. (Left): typical infrared thermal imaging pictures after 5 min heating under $M H T$ alone $(110 \mathrm{kHz}, 25 \mathrm{mT})$, PLASM alone $\left(680 \mathrm{~nm}, 0.3 \mathrm{~W} / \mathrm{cm}^{2}\right)$ and bimodal MHT+PLASM (same parameters). (Right) Typical temperature elevation profiles of the injected $(\mathrm{T}+$ and non-injected tumours ( $\mathrm{T}-$, control tumour) as a function of time for the three treatments.

\section{Conclusions}

To summarize, we have demonstrated that an adequate magnetoplasmonic design can efficiently combine photothermia with magnetic hyperthermia into an efficient bimodal thermo-therapy. The proposed stable and biocompatible magneto-plasmonic nanohybrids were composed of an iron oxide multi-core optimized for high efficiency in magnetic hyperthermia, and a gold shell with tunable plasmonic properties in the NIR region. We provided nanoscale characterization by mapping for the first time the plasmon around a magnetic oxide core, connecting unambiguously the optical response of single, isolated nanostructures with that of large assemblies. We then demonstrated the versatility of the system in generating heat through a remarkable cumulative effect when both magnetic and plasmonic modalities were applied simultaneously and showed that heating efficiency was maintained in in vivo conditions. The tumor temperature can then rapidly reach $48^{\circ} \mathrm{C}$, condition for therapeutic tumor ablation, with dose injected 10 times lower than for classical magnetic hyperthermia treatment. $^{29}$

A. E. acknowledges support from the European Commission under a Marie Curie Intra-European project FP7-PEOPLE-2013-IEF-626471. G.R. acknowledges Institut de Physique (INP) of Centre National de la Recherche Scientifique (CNRS) for partial financial through a PICS. G. A. B. is grateful to the Natural Sciences and Engineering Research Council (NSERC), Canada, for a Discovery grant partially supporting this work. The STEM and EELS work was carried at the Canadian Centre for Electron Microscopy, a national facility supported by NSERC, the Canada Foundation for Innovation and McMaster University. A. A. H acknowledges N. Menguy (IMPMC lab, UPMC) for discussions. We thank A. Djemat from Buffon Animalery for animal care and housing.

\section{Notes and references}

1. (a) V. Wagner, A. Dullaart, A.-K. Bock and A. Zweck, Nat. Biotech, 2006, 24, 1211-1217; (b) K. Riehemann, S. W. Schneider, T. A. Luger, B. Godin, M. Ferrari and H. Fuchs, Angew. Chem. Int. Ed., 2009, 48, 872-897.

2. (a) C. S. Levin, C. Hofmann, T. A. Ali, A. T. Kelly, E. Morosan, P. Nordlander, K. H. Whitmire and N. J. Halas, ACS Nano, 2009, 3, 1379-1388; (b) R. Di Corato, G. Béalle, J. Kolosnjaj-Tabi, A. Espinosa, O. Clément, A. K. Silva, C. Menager and C. Wilhelm, ACS Nano, 2015, 9, 2904-2916.

3. (a) M. Colombo, S. Carregal-Romero, M. F. Casula, L. Gutiérrez, M. P. Morales, I. B. Böhm, J. T. Heverhagen, D. Prosperi and W. J. Parak, Chem. Soc. Rev., 2012, 41, 4306-4334; (b) R. Di Corato, A. Espinosa, L. Lartigue, M. Tharaud, S. Chat, T. Pellegrino, C. Ménager, F. Gazeau and C. Wilhelm, Biomaterials, 2014, 35, 6400-6411; (c) P. K. Jain, K. S. Lee, I. H. El-Sayed and M. A. ElSayed, J. Phys. Chem. B, 2006, 110, 7238-7248.

4. (a) X. Huang, I. H. El-Sayed, W. Qian and M. A. El-Sayed, J. Am. Chem. Soc., 2006, 128, 2115-2120; (b) M. Garcia, J. Phys. D: Appl. Phys., 2011, 44, 283001.

5. (a) H. Jans and Q. Huo, Chem. Soc. Rev., 2012, 41, 2849-2866; (b) A. M. Gobin, M. H. Lee, N. J. Halas, W. D. James, R. A. Drezek and J. L. West, Nano Lett., 2007, 7, 1929-1934.

6. R. Bardhan, S. Lal, A. Joshi and N. J. Halas, Acc. Chem. Res., 2011, 44, 936-946.

7. (a) X. Wang, G. Li, Y. Ding and S. Sun, RSC Adv., 2014, 4, 3037530383; (b) H. Yuan, C. G. Khoury, C. M. Wilson, G. A. Grant, A. J. Bennett and T. Vo-Dinh, Nanomedicine, 2012, 8, 1355-1363.

8. (a) X. Huang, S. Neretina and M. A. El-Sayed, Adv. Mat., 2009, 21, 4880-4910; (b) X.-M. Zhu, C. Fang, H. Jia, Y. Huang, C. H. Cheng, C.-H. Ko, Z. Chen, J. Wang and Y.-X. J. Wang, Nanoscale, 2014, 6, 11462-11472.

9. E. C. Dreaden, A. M. Alkilany, X. Huang, C. J. Murphy and M. A. El-Sayed, Chem. Soc. Rev., 2012, 41, 2740-2779. 
10. P. Guardia, R. Di Corato, L. Lartigue, C. Wilhelm, A. Espinosa, M. Garcia-Hernandez, F. Gazeau, L. Manna and T. Pellegrino, ACS Nano, 2012, 6, 3080-3091.

11. L. Lartigue, P. Hugounenq, D. Alloyeau, S. P. Clarke, M. Lévy, J.C. Bacri, R. Bazzi, D. F. Brougham, C. Wilhelm and F. Gazeau, ACS Nano, 2012, 6, 10935-10949.

12. J.-H. Lee, J.-t. Jang, J.-s. Choi, S. H. Moon, S.-h. Noh, J.-w. Kim, J.-G. Kim, I.-S. Kim, K. I. Park and J. Cheon, Nat. Nanotech., 2011, 6, 418-422.

13. (a) J. Gao, H. Gu and B. Xu, Acc. Chem. Res., 2009, 42, 10971107; (b) A. K. Gupta and M. Gupta, Biomaterials, 2005, 26, 3995-4021; (c) M. Hu, J. Chen, Z.-Y. Li, L. Au, G. V. Hartland, X. Li, M. Marquez and Y. Xia, Chem. Soc. Rev., 2006, 35, 10841094.

14. (a) T. A. Larson, J. Bankson, J. Aaron and K. Sokolov, Nanotechnology, 2007, 18, 325101; (b) C.-H. Wu, J. Cook, S. Emelianov and K. Sokolov, Adv. Funct. Mat., 2014, 24, 68626871; (c) I. Urries, C. Muñoz, L. Gomez, C. Marquina, V. Sebastian, M. Arruebo and J. Santamaria, Nanoscale, 2014, 6, 9230-9240; (d) A. J. Coughlin, J. S. Ananta, N. Deng, I. V. Larina, P. Decuzzi and J. L. West, Small, 2014, 10, 556-565; (e) G. A. Sotiriou, F. Starsich, A. Dasargyri, M. C. Wurnig, F. Krumeich, A. Boss, J.-C. Leroux and S. E. Pratsinis, Adv. Funct. Mat., 2014, 24, 2818-2827; (f) Z. Fan, M. Shelton, A. K. Singh, D. Senapati, S. A. Khan and P. C. Ray, ACS Nano, 2012, 6, 1065-1073; (g) M. Masoumi, Y. Wang, M. Liu, M. Tewolde and J. Longtin, 2015; (h) D. K. Kirui, D. A. Rey and C. A. Batt, Nanotechnology, 2010, 21, 105105; (i) F. Mohammad, G. Balaji, A. Weber, R. M. Uppu and C. S. Kumar, J. Phys. Chem. C, 2010, 114, 19194-19201.

15. K. M. L. Taylor-Pashow, J. Della Rocca, R. C. Huxford and W. Lin, Chem. Commun., 2010, 46, 5832-5849.

16. M. C. Daniel and D. Astruc, Chem. Rev., 2004, 104, 293-346.

17. Y. Javed, L. Lartigue, P. Hugounenq, Q. L. Vuong, Y. Gossuin, R. Bazzi, C. Wilhelm, C. Ricolleau, F. Gazeau and D. Alloyeau, Small, 2014, 10, 3325-3337.

18. (a) M. Abdulla-Al-Mamun, Y. Kusumoto, T. Zannat, Y. Horie and H. Manaka, RSC Advances, 2013, 3, 7816-7827; (b) S. Balasubramanian, A. R. Girija, Y. Nagaoka, T. Fukuda, S. Iwai, V. Kizhikkilot, K. Kato, T. Maekawa and S. D. Nair, RSC Advances, 2015, 5, 25066-25078.

19. P. Quaresma, I. Osorio, G. Doria, P. A. Carvalho, A. Pereira, J. Langer, J. P. Araujo, I. Pastoriza-Santos, L. M. Liz-Marzan, R. Franco, P. V. Baptista and E. Pereira, RSC Adv., 2014, 4, 36593667.

20. P. Hugounenq, M. Levy, D. Alloyeau, L. Lartigue, E. Dubois, V. Cabuil, C. Ricolleau, S. Roux, C. Wilhelm, F. Gazeau and R. Bazzi, J. Phys. Chem. C., 2012, 116, 15702-15712.

21. B. Broennum, H. S. Johansen and L. Skibsted, Inorg. Chem., 1988, 27, 1859-1862.

22. S. Barbosa, A. Agrawal, L. Rodríguez-Lorenzo, I. PastorizaSantos, R. A. Alvarez-Puebla, A. Kornowski, H. Weller and L. M. Liz-Marzán, Langmuir, 2010, 26, 14943-14950.

23. E. J. Kirkland, Adv. Comp. Elect. Microscopy, Springer, 2010.

24. J. Lim, A. Eggeman, F. Lanni, R. D. Tilton and S. A. Majetich, Adv. Mat., 2008, 20, 1721-1726.

25. Z. Xu, Y. Hou and S. Sun, J. Am. Chem. Soc., 2007, 129, 86988699.

26. S. Prabha, W.-Z. Zhou, J. Panyam and V. Labhasetwar, Int. J. Pharm., 2002, 244, 105-115.

27. (a) J. Nelayah, M. Kociak, O. Stéphan, F. J. G. de Abajo, M. Tencé, L. Henrard, D. Taverna, I. Pastoriza-Santos, L. M. LizMarzán and C. Colliex, Nat. Phys., 2007, 3, 348-353; (b) D.
Rossouw and G. A. Botton, Phys. Rev. Lett., 2013, 110, 066801; (c) O. Nicoletti, F. de La Peña, R. K. Leary, D. J. Holland, C. Ducati and P. A. Midgley, Nature, 2013, 502, 80-84.

28. B. V. Robinson, F. M. Sullivan, J. F. Borzelleca and S. L. Schwartz, PVP: A Critical Review of the Kinetics and Toxicology of Polyvinylpyrrolidone (Povidone), Lewis Publishers; 1 edition (May 9, 1990), 1990.

29. (a) M. Johannsen, U. Gneveckow, B. Thiesen, K. Taymoorian, C. H. Cho, N. Waldöfner, R. Scholz, A. Jordan, S. A. Loening and P. Wust, Eur. Urol., 2007, 52, 1653-1662; (b) K. Maier-Hauff, F. Ulrich, D. Nestler, H. Niehoff, P. Wust, B. Thiesen, H. Orawa, V. Budach and A. Jordan, J. Neuro-Oncol., 2011, 103, 317-324. 